\title{
Orquitis granulomatosa idiopática bilateral
}

\author{
Peyrí Rey E, Riverola Manzanilla A*, Cañas Tello MA*. \\ Servicio de Urología. *Servicio de Histopatología. Clínica Delfos. Barcelona.
}

Actas Urol Esp. 2008;32(4):461-463

\section{RESUMEN}

ORQUITIS GRANULOMATOSA IDIOPATICA BILATERAL

Describimos un raro caso de sincronismo asintomático de orquitis idiopática granulomatosa bilateral. La ecografia escrotal muestra múltiples áreas hipocóicas, el diagnóstico diferencial entre tumor y orquitis granulomatosa es muy difícil y sólo el examen histológico lo confirmará.

Palabras clave: Granuloma. Orquitis. Idiopática. Bilateral.

\section{ABSTRACT \\ GRANULOMATOUS ORCHITIS IDIOPATHIC BILATERAL}

A rare case of asymtomatic synchronous bilateral granulomatous orchitis idiopathic is decribed. In the scrotal ultrasonography are multiple hypoecoic areas, differential diagnosis between testicular tumor and granulomatous orchitis is very difficult in any examination by histological findings.

Keywords: Granuloma. Orchitis. Idiopathic. Bilateral.

$\mathrm{L}$ a orquitis granulomatosa idiopática es una rara lesión inflamatoria de etiología desconocida o incierta, desde el punto de vista clínico es importante por ser su presentación semejante a un tumor testicular. La combinación de la historia clínica junto el estudio de laboratorio y la ecografía nos pueden dar el diagnóstico ${ }^{3,11,13}$. Nosotros presentamos un raro caso de orquitis granulomatosa idiopática con afectación sincrónica de ambos testículos, lesión bilateral con sólo 4 casos publicados en la literatura ${ }^{4,6,7,10}$.

\section{CASO CLÍNICO}

Varón de 75 años de edad, con insuficiencia respiratoria, sin otros antecedentes personales de interés. Acude por presentar una escrotomegalia bilateral, mayor derecha asintomática, de 6 meses de evolución, tratada con antibióticos e inflamatorios. A la exploración clínica presenta una escrotomegalia bilateral indolora con transiluminación positiva, palpándose nódulos duros testiculares bilaterales, mayor el derecho, el epidídimo izquierdo es duro, difícil de palpar el derecho. Se excluye el diagnóstico de tuberculosis, mediante radiografía de tórax y test de Mantoux y Elisa negativos. Analítica de sangre y de orina normal alfafetoproteína $2 \mathrm{ng} / \mathrm{ml}$ y bhcg $2,6 \mathrm{ml} \mu / \mathrm{ml}$. Ecografía escrotal (Fig. 1) con hidrocele bilateral mayor el derecho con proyección y extensión hacia canal inguinal. El testículo derecho esta aumentado de tamaño, apreciándose en su interior una masa globalmente hipocogénica si bien con áreas de diferente ecogenidad de 47x27 mm. En el testículo izquierdo se visualiza un área hipoecogénica de $24 \mathrm{~mm}$, visualizando el epidídimo con ecogenidad alterada. Practicó orquiectomía derecha vía inguinal y posteriormente biopsia del testículo izquierdo, después de descartar un proceso maligno.

El análisis histológico presenta microscópicamente una lesión nodular con área quística. Microscópicamente (Fig. 2) parénquima testicular con proceso inflamatorio crónico de tipo granulomatoso, con área de necrosis central rodeada de granulomas constituidos principalmente por histiocitos, en menor proporción células gigantes multinucleadas y linfocitos. El parénquima testicular adyacente a la lesión presenta cambios atróficos, la tinción de pas y ziehl-neelsen para la detección de 


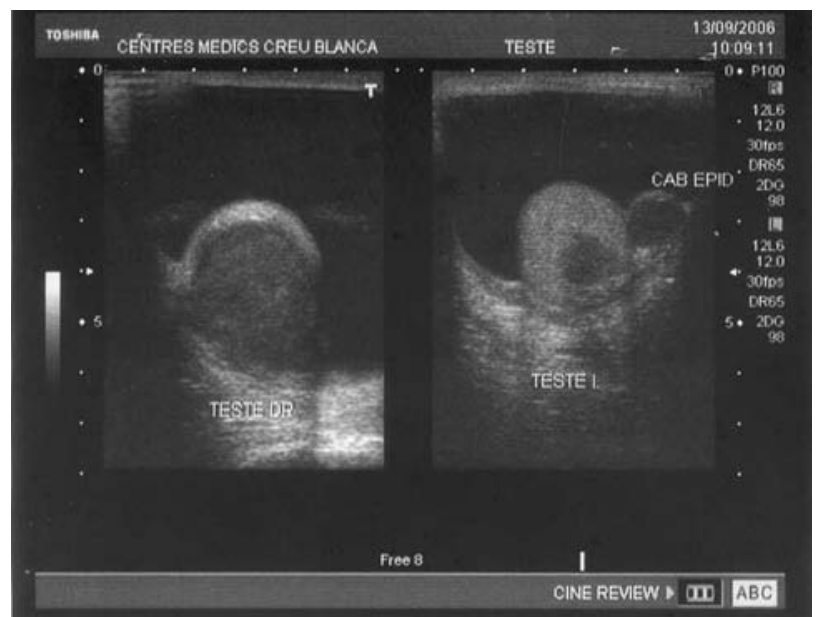

FIGURA 1: Ecografia testicular que muestra hidrocele bilateral, masa hipoecogénica de $47 \times 27 \mathrm{~mm}$ en el testículo derecho y área hipoecogénica de $24 \mathrm{~mm}$ en el izquierdo, junto con alteración ecogénica heterogénea en el epididimo izquierdo.

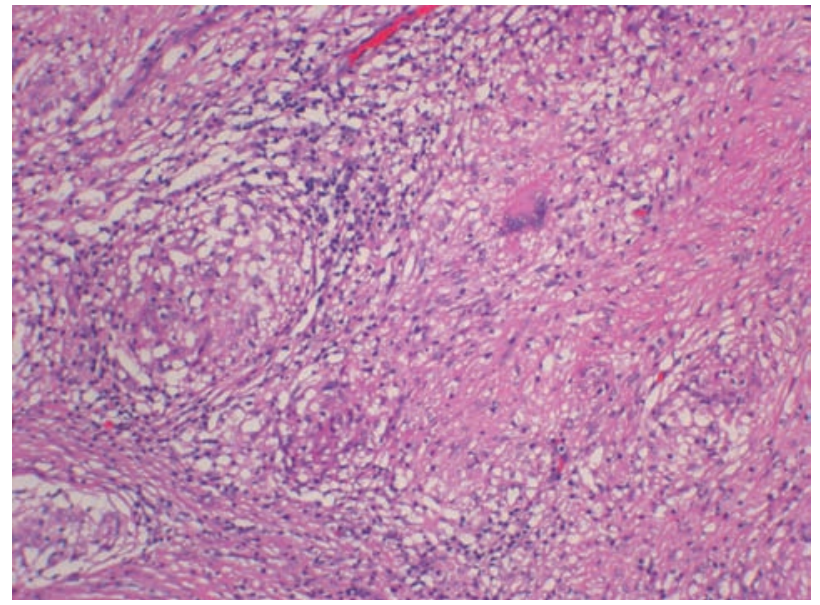

FIGURA 2: Histologia, que muestra granuloma, con necrosis central, histiocitos con células gigante multinucleadas, observando periféricamente linfocitos.

microorganismos ha resultado negativa. Posteriormente se le propone al enfermo la orquiectomía contralateral, prefiriendo el paciente el control evolutivo.

\section{DISCUSIÓN}

La orquitis granulomatosa puede ser de etiología específica, o idiopática que es la etiología más rara. La tuberculosis es la causa más frecuente de las especifícas, seguida de la brucelosis, sífilis, actinomicosis y la sarcoidosis $3,9,14,16$. El granuloma afectando al epidídimo, es más frecuente en la tuberculosis, en el que los granulomas tienen necrosis de caseificación central, que después de afectar el epidídimo se prolongan al testículo. Está descrita la OG como complicación tras la administración de BCG intravesical en el tratamiento del tumor vesical ${ }^{14}$. La afectación granulomatosa por brucelosis, lepra y sífilis, suelen tener el antecedente previo de padecer dichas enfermedades. En la sarcoidosis, aparte de acompañarse de compromiso ganglionar, si se involucra el testículo, los granulomas epiteloides sin caseosis incluyen células gigantes multinucleadas con cuerpos asteroides y basófilos de Schauman ${ }^{16}$.

La orquitis granulomatosa idiopática es una inflamación normalmente crónica del parénquima testicular. De etiología desconocida, se manejan como posibles causas primeramente el traumatismo previo sobre el testículo (parece que puede conducir a una insuficiencia vascular). La segunda causa apunta a una posible reacción inmunológica del esperma; la reacción granulomatosa se produce en respuesta a una inflamación secundaria a la extravasación de esperma contenido en los túbulos seminíferos. El papel de la infección en la etiología de la OG idiopáticas no debe ser olvidado. Se ha encontrado infección bacteriana del tracto urinario, en pacientes con OG (hasta el 30\% de los casos en alguna serie), pero no se ha podido demostrar directamente su relación ${ }^{3,11,13}$.

Descrita por primera vez por Grunberg en $1926^{1}$, suele presentarse unilateralmente en varones de 19 a 84 años (más frecuente 50-70 años), aunque hay descrito un caso en un niño de 8 años ${ }^{2}$.

Los pacientes con OG suelen presentar molestias leves o nula de larga evolución, acompañado de aumento o tumefacción testicular, algún caso presento dolor escrotal agudo ${ }^{8,9,11}$. La exploración física de los testículos afectados normalmente no permite la diferenciación entre un tumor testicular o con una posible orquitis granulomatosa. No se ha detectado ningún agente causante de la OG en cultivos o tinciones especiales. La ecografía tan efectiva en la patología del contenido escrotal, no es útil en el diagnóstico diferencial con los tumores malignos. No obstante la OG suele describirse como testículos difusamente hipocoicos con áreas focales intratesticulares pobre o bien definidas ${ }^{4,5,14}$. La histología suele presentar dos patrones anatomopatológicos característicos:

1. Inflamación crónica del intersticio con infiltrado, predominantemente de linfocitos y células plasmáticas con presencia usual de células gigantes. 
2. Destrucción de las células germinales en los túbulos seminíferos.

En nuestro caso el hecho de que los marcadores tumorales fueran negativos, junto con el hecho de ser la afectación testicular bilateral sincrónica, junto con la ecografía (lesión de un epidídimo) nos hizo sospechar que no se tratase de una neoplasia testicular.

El tratamiento conservador con corticoides, antinflamatarios o antibióticos no es efectivo. La orquiectomía es el único tratamiento efectivo. No parece existir relación entre la OG y el carcinoma testicular, no se ha descrito ningún caso de OG que presentase simultánea o posteriormente neoplasia testicular $8,11,15$.

\section{REFERENCIAS}

1. Grünberg H. Three unusual cases of chronic orchitis clynically resembling tumors of the testis.Frankfurt $Z$ the Pathol. 1926; 33:217-27.

2. Nishizawa S,Hashimoto S,Shiyama A et al.Granulomatous orchitis in a child. B J U International. 2000:85(6):776.

3. Osca Garcia JM,Alfaro Ferreres 1, Ruiz Cerda JL et al. Orquitis granulomatosa idiopática. Actas Urol Esp. 1993;16(1):53-56.

4. Pekindil G, Hüseyin Atakan I, Kaya E, Bilgi S, Inci O. Bilateral synchronous granulomatous orchitis gray-scale and colour doppler sographic findings. Eur J Radiol. 1999;31(3):201-203.

5. MogensenM,Nino M. Idiopathic granulomatous epiddymo-orquitis: sonographic appearance. J Ultrasound Med. 2005;24 (7); 107-110

6. Cocimano V, Marino G, Torchio B, Brigato R. A rare case bilateral granulomatous orchitis.Minerva Urol Nefrol. 1989:41(4) 283-286.
7. Okajima E, Cho M, Maruyama Y. Asymtomatic synchronous bilateral granulomatous orchitis.a case report. Hinyokika Kiyo. 1994;40(12).1123-1126.

8. Martínez-Rodríguez M, Navarro Fos S, Soriano Sarrió P, AlcaláSantaella Casanova C, Ramos Soler D, et al. Orquitis granulomatosa. Arch Esp Urol. 2006;59(7):725-727.

9. Sakuma T, Deu S. A case report of granulomatous orchitisreview of 20 cases in japon. Nippon Hntokika Gakkai Zasshi. 2001;92(1):30-33

10. Rubins I. Bilateral granulomatous orchitis: manifestation of idiopathic systemic granulomatosis. J Urol. 1987;137(2):317.

11. Fauer RB, Goldstein AM, Green JC, Onofrio R. Clinical aspects of granulomatous orchitis. Urology.1978;12(4):416-419.

12. Perimenis P, Athanasopoulos A, Venetsanou-Petrochilou C, Barbalias G. Idiopathic granulomatous orchitis. Eur Urol. 1991;19(2):118-120.

13. Wegner HE, Loy V, Dieckmann KF. Granulomatous orchitis-an analysis of clinical presentation, pathological anatomic features and posible etiologic factors. Eur Urol. 1994;26(1):56-60.

14. Salmeron I, Ramirez-Escobar MA, Puertas F, Marcos R, GarciaMarcos F, Sanchez R. Granulomatous epididymo-orchitis: sonographic features and clinical outcome in brucellosis, tuberculosis and idiopathic granulomatous epididymo-orchitis. J Urol. 1998;159(6):1954-1957.

15. Kisbedek L,Nemeth A Granulomatous orchitis and spermatic granuloma.Intenational Urology and Nephrology. 1975;7(2); 141-148.

16. Calleja Escudero J, de la Cruz Ruíz M, Rivera Ferro J, Torrecilla García-Ripoll JR, Peral Martínez JI, Fernández del Busto E. Orquitis granulomatosa. Actas Urol Esp. 2000;24(8): 682-684.

Correspondencia autor: Dr. E. Peyrí Rey

Servicio de Urología. Clínica Delfos

Avda. Vallcarca, 149-151 Barcelona. Tel.: 932156202

E-mail autor: 7956epr@comb.cat

Información artículo: Nota Clínica

Trabajo recibido: diciembre 2006

Trabajo aceptado: enero 2007 